\title{
Comparación de la composición corporal entre veganos y ovolactovegetarianos en Aguascalientes
}

Martínez Medina Jennifer Nazat*, González Muñoz María Paulina*, Merino Méndez Alejandra*, Ruvalcaba García Carolina*, Huerta Andrea Del Carmen*, Palomar Barrientos Claudia Alejandra*, Sánchez Macías Andrea*, Martín del Campo Cervantes Judith**

\begin{tabular}{|c|c|}
\hline $\begin{array}{l}\text { Resumen } \\
\text { Introducción: Los principales componentes del cuerpo humano } \\
\text { son masa magra (MM), masa grasa (MG) y porcentaje de } \\
\text { agua, los cuales son poco conocidos entre los vegetarianos, } \\
\text { mismos que presentan un menor índice de masa corporal } \\
\text { (IMC) en comparación con las personas que sí consumen } \\
\text { carne. Objetivo: Comparar la composición corporal de adultos } \\
\text { sanos veganos y ovolactovegetarianos de } 20 \text { a } 30 \text { años de la } \\
\text { ciudad de Aguascalientes de agosto a noviembre del } 2014 \text {. } \\
\text { Material y Métodos: Observacional, descriptivo transeccional y } \\
\text { transversal, donde se estudiaron a } 60 \text { vegetarianos (30 ovo- } \\
\text { lactovegetarianos y } 30 \text { veganos), seleccionados de forma no } \\
\text { probabilística por cuota; a los cuales se les tomaron medicio- } \\
\text { nes antropométricas (peso y estatura) y de composición cor- } \\
\text { poral como masa grasa total (MGT en \%) y masa magra total } \\
\text { (MMT en kg). Se usó estadística descriptiva y T-Student para la } \\
\text { comparación de medias entre los dos grupos. Resultados: En } \\
\text { el presente estudio se obtuvo una diferencia significativa entre } \\
\text { el grupo de los ovolactovegetarianos y los veganos del género } \\
\text { masculino en el IMC (kg/m2) (p = 0.006), en el \% de MGT } \\
\text { (p = 0.0 I) y en los kg de MMT (p = 0.03), donde los valores } \\
\text { fueron mayores en el grupo de los ovolactovegetarianos, no } \\
\text { obteniendo los mismos resultados para el género femenino. } \\
\text { Conclusiones: Los ovolactovegetarianos del género masculino } \\
\text { presentan mayor IMC, MGT y MMT que los veganos. LUX MÉ- } \\
\text { DICA, AÑo 10, NúmERO31,SEPTIEMBRE-DICIEMBRE 2015, PP 11-16 }\end{array}$ & 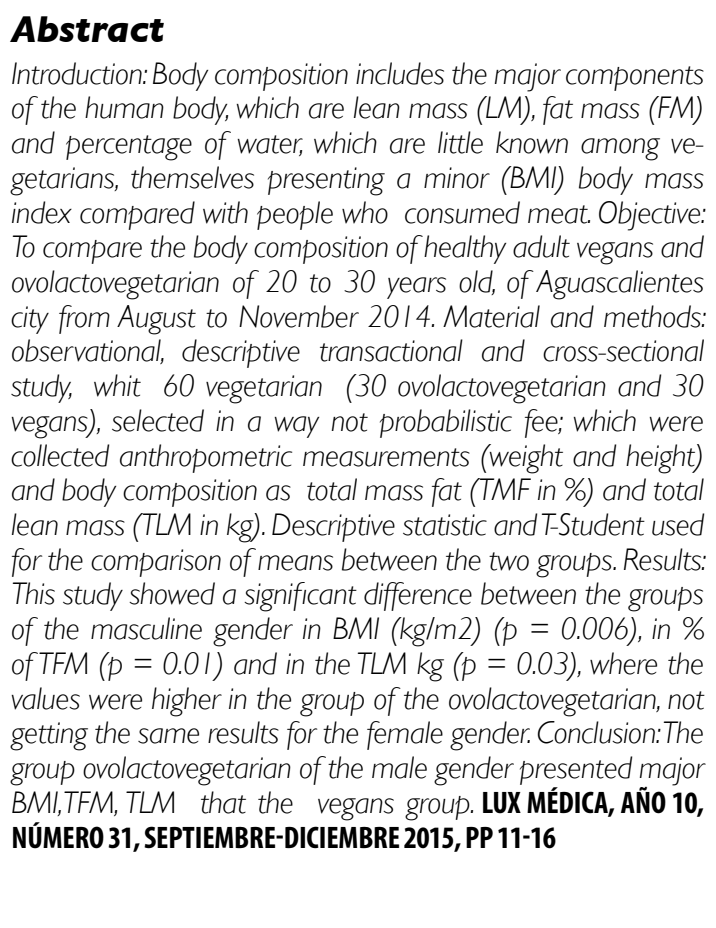 \\
\hline $\begin{array}{r}\text { Palabras clave: Composición corporal, ovolactovegeta- } \\
\text { rianos, veganos, vegetarianos, masa magra total y masa } \\
\text { grasa total. }\end{array}$ & $\begin{array}{l}\text { Key words: body composition, ovolactovegetarian, vegan, } \\
\text { vegetarian, total lean mass and total fat mass. }\end{array}$ \\
\hline
\end{tabular}

* Estudiantes de la Licenciatura en Nutrición del Centro de Ciencias de la Salud de la Universidad Autónoma de Aguascalientes.

** Profesora investigadora de la Licenciatura de Nutrición del Centro de Ciencias de la Salud de la Universidad Autónoma de Aguascalientes.

Fecha de recibido: 10 de agosto de 2015

Fecha de aceptación: 15 de septiembre de 2015

Correspondencia: Judith Martín del Campo, Departamento de Nutrición y Cultura Física, Centro de Ciencias de la Salud, Universidad Autónoma de Aguascalientes. Avenida Universidad. Número 940. Ciudad Universitaria. Código postal 20131. Aguascalientes, Aguascalientes., México. Teléfono +52(449)9108443. Correo electrónico jmartic@correo.uaa.mx 


\section{Introducción}

Los efectos de los diferentes tipos de dietas han sido muy estudiados; la dieta vegetariana está tomando importancia en la actualidad por varios motivos como los relacionados con la salud, los éticos y los ecológicos. Según la Unión Vegetariana Internacional se define al vegetarianismo como "la práctica de no comer carne roja, pollo o pescado ni sus derivados; con la posibilidad de incluir o no el consumo de huevos o productos lácteos". Estudios epidemiológicos y clínicos clasifican a los vegetarianos en veganos, lactovegetarianos y ovolactovegetarianos ${ }^{1-3}$.

La Asociación Dietética Americana menciona que las dietas vegetarianas bien planificadas son saludables, nutricionalmente adecuadas y proporcionan beneficios a la salud ${ }^{4}$.

Por otro lado, se conoce que la composición corporal es la suma de los compartimientos de masa grasa (MG), masa magra (MM) y porcentaje de agua, los cuales pueden verse modificados por la dieta. La masa magra difiere entre los géneros, siendo en los adultos del $70 \%$ al $75 \%$ de este compartimiento agua corporal. Mientras que el de la masa grasa es el más variable debido a los cambios que presenta una persona desde la niñez hasta la vejez ${ }^{5-9}$.

De acuerdo a Brignardello G.J. encontró que los vegetarianos son, en promedio, más delgados que los no vegetarianos, así mismo Key T.J., muestra que los vegetarianos tienen un IMC de $1 \mathrm{~kg} /$ $\mathrm{m} 2$ menor que los no vegetarianos ${ }^{1,10}$.

Debido a lo anterior se pretende comparar la diferencia en la composición corporal de adultos sanos veganos y ovolactovegetarianos que se encuentren en un rango de edad de 20 a 30 años, que habiten en la ciudad de Aguascalientes.

\section{| | | | | | | | | | | | | | | | | | | | | | | | | | | | | | | | | | | | | | | | | | | | | | | | | | | | | | | | | | | | | | | | | | | | | | | | | | | | | | | | | | | | | | | | | | | | | | | | | | | | |}

\section{Material y métodos}

Estudio observacional, descriptivo transeccional y transversal con una población de 60 vegetarianos (30 ovolactovegetarianos y 30 veganos) con un rango de edad de 20 a 30 años. La técnica de muestreo fue no probabilístico por cuota. Para el análisis de la composición corporal, se utilizó la báscula de bioimpedancia Tanita Ironman Inner Scan (frecuencia de $50 \mathrm{kHz}, 500 \mu \mathrm{A}$, capacidad de $150 \mathrm{~kg}$ ) para obtener la masa magra total en $\mathrm{kg}(\mathrm{MMT})$, el porcentaje de masa grasa total (\% MGT) y el porcentaje de agua corporal total (\% ACT). Para obtener la talla, se utilizó el estadímetro portátil
SECA 214 (rango de medición de $20 \mathrm{~cm}$ a $207 \mathrm{~cm}$, precisión de $1 \mathrm{~mm}$ ).

La población fue medida con ropa ligera, mínimo con 4 horas de ayuno, con la vejiga vacía, sin haber realizado ejercicio físico intenso $24 \mathrm{~h}$ antes a la medición y sin objetos metálicos que pudieran afectar los valores estimados por el equipo de la bioimpedancia eléctrica (BIA). Se realizó la estadística con el programa IBM SPSS Statistics versión 20 para la diferencia de medias mediante t-Student. El nivel de significancia que se aceptó fue de $p<0.05$, con un intervalo de confianza de $95 \%$. 


\section{Resultados}

Se estudiaron a 60 personas vegetarianas de la cuales el $45 \%$ fueron hombres (27 pacientes) y el $55 \%$ fueron mujeres (33 pacientes), con una edad promedio de 24,2 años ( $D E \pm 3,6$ años). El grupo de los ovolactovegetarianos tuvo una distribución del $60 \%$ del género femenino $(n=18)$ y el $40 \%$ del género mas- culino ( $n=12$ ), mientras que en el grupo de los veganos se tuvo una distribución del $50 \%$ de cada género.

Se realizaron cuestionamientos a los dos grupos acerca del cambio de peso y tipo de cambio corporal por el hecho de ser vegetarianos, así como la realización de actividad física, mismos que se presentan en la tabla No. 1.

\section{Tabla I}

Cambio corporal, tipo de cambio corporal y actividad física según el tipo de vegetarianismo.

\begin{tabular}{|c|c|c|c|}
\hline Tipo de vegetarianismo & Variable & Opciones & Porcentaje (n) \\
\hline \multirow{8}{*}{ Ovolactovegetariano } & \multirow{3}{*}{ Cambio corporal } & Sí & $53.3(16)$ \\
\hline & & No & $46.7(14)$ \\
\hline & & Aumento de peso & $3.3(1)$ \\
\hline & \multirow[t]{4}{*}{ Tipo de cambio } & Disminución de peso & $33.3(10)$ \\
\hline & & Otro & $13.3(4)$ \\
\hline & & No referido & $50(15)$ \\
\hline & & Sí & $76.7(23)$ \\
\hline & Realiza actividad física & No & $23.3(7)$ \\
\hline \multirow{8}{*}{ Vegano } & \multirow{3}{*}{ Cambio corporal } & Sí & $76.7(23)$ \\
\hline & & No & $23.3(7)$ \\
\hline & & Aumento de peso & $3.3(1)$ \\
\hline & \multirow{3}{*}{ Tipo de cambio } & Disminución de peso & $40.0(12)$ \\
\hline & & Otro & $30.0(9)$ \\
\hline & & No referido & $26.7(8)$ \\
\hline & \multirow{2}{*}{ Realiza actividad física } & Sí & $76.7(23)$ \\
\hline & & No & $23.3(7)$ \\
\hline
\end{tabular}

$n=60$ vegetarianos (30 veganos y 30 ovolactovegetarianos).

Al evaluar los datos antropométricos se obtuvieron los promedios para la talla, el peso, el IMC y el ACT los cuales fueron para el grupo de ovolactovegetarianos de $1,66 \mathrm{~m}(\mathrm{DE} \pm 0,09 \mathrm{~m}), 64,89 \mathrm{~kg}(\mathrm{DE} \pm$ $12,84 \mathrm{~kg}), 23,28 \mathrm{~kg} / \mathrm{m}^{2}\left(\mathrm{DE} \pm 2,78 \mathrm{~kg} / \mathrm{m}^{2}\right)$ y $54,5 \%$ (DE $\pm 4,92 \%$ ) respectivamente; mientras que para el grupo de los veganos fueron de 1,66 $\mathrm{m}(\mathrm{DE} \pm 0,08 \mathrm{~m}), 61,17 \mathrm{~kg}$ $(D E \pm 7,28 \mathrm{~kg}), 21,99 \mathrm{~kg} / \mathrm{m}^{2}(\mathrm{DE} \pm 2,44$ $\left.\mathrm{kg} / \mathrm{m}^{2}\right)$ y $57,1 \%(\mathrm{DE} \pm 5,66 \%)$.
En cuanto a la comparación de medias del IMC según el tipo de vegetarianismo entre los hombres de ambos grupos se obtuvo una diferencia significativa $(p=0,006)$; mientras que para las mujeres no se encontró diferencia $(p=0,98)$, en la tabla 2 se describen los datos según los grupos de vegetarianos y el género.

La composición corporal se evaluó mediante el BIA, donde se obtuvieron los siguientes datos: el promedio de la MMT en 
hombres ovolactovegetarianos fue de 57,5 $\mathrm{kg}$ (DE $\pm 7 \mathrm{~kg}$ ) y la de los veganos fue de $51,79 \mathrm{~kg}$ (DE $\pm 6,11 \mathrm{~kg})$, mientras que los ovolactovegetarianos hombres tuvieron un promedio en la MGT de $18,84 \%$ (DE \pm $4,87 \%$ ) y en los veganos fue de $13,86 \%$ $(\mathrm{DE} \pm 4,47 \%$ ); la tabla 2 muestra los datos de acuerdo al tipo de vegetarianismo y el género de los sujetos. Al comparar los dosgrupos se obtuvo una diferencia significativa entre los hombres veganos y los ovolactovegetarianos en el IMC ( $p=0,006)$, en la MMT $(p=0,03)$ y en la MGT ( $p=$ 0,01). En la comparación entre mujeres de ambos grupos no se encontraron diferencias significativas.

\section{Tabla 2}

Datos de la comparación de medias en la composición corporal tomados mediante BIA y el IMC.

\begin{tabular}{|c|c|c|c|c|c|c|}
\hline \multicolumn{2}{|l|}{ Género } & \multirow{2}{*}{$\begin{array}{l}\text { Tipo de vegetariano } \\
\text { Ovolactovegetariano }\end{array}$} & \multirow{2}{*}{$\begin{array}{c}\mathbf{n} \\
18\end{array}$} & \multirow{2}{*}{$\begin{array}{l}\text { Media } \\
22.45\end{array}$} & \multirow{2}{*}{$\begin{array}{c}\text { DE } \\
2.71\end{array}$} & \multirow{2}{*}{$\begin{array}{r}\mathbf{p} \\
0.98\end{array}$} \\
\hline & IMC & & & & & \\
\hline & $(\mathrm{kg} / \mathrm{m} 2)$ & Vegano & 15 & 22.47 & 2.24 & \\
\hline \multirow{4}{*}{ Femenino } & MMT & Ovolactovegetariano & 18 & 39.49 & 4.31 & \multirow{2}{*}{0.57} \\
\hline & $(\mathrm{kg})$ & Vegano & 15 & 40.19 & 2.17 & \\
\hline & MGT & Ovolactovegetariano & 18 & 28.34 & 5.90 & \multirow{2}{*}{0.41} \\
\hline & $(\%)$ & Vegano & 15 & 26.72 & 5.09 & \\
\hline \multirow{6}{*}{ Masculino } & IMC & Ovolactovegetariano & 12 & 24.52 & 2.49 & \multirow{2}{*}{0.006} \\
\hline & $(\mathrm{kg} / \mathrm{m} 2)$ & Vegano & 15 & 21.51 & 2.62 & \\
\hline & MMT & Ovolactovegetariano & 12 & 57.50 & 7.00 & \multirow{2}{*}{0.03} \\
\hline & $(\mathrm{kg})$ & Vegano & 15 & 51.79 & 6.11 & \\
\hline & MGT & Ovolactovegetariano & 12 & 18.84 & 4.87 & \multirow{2}{*}{0.01} \\
\hline & $(\%)$ & Vegano & 15 & 13.86 & 4.47 & \\
\hline
\end{tabular}

$n=60$ vegetarianos (30 veganos y 30 ovolactovegetarianos)

$I M C=$ Índice de masa corporal, $M M T=$ Masa magra total, $M G T=$ Masa grasa total y DE= desviación estándar

\section{Discusión}

Se encontró que la población de veganos presentó un IMC menor $\left(21,99 \mathrm{~kg} / \mathrm{m}^{2}\right)$ en comparación a la población de ovolactovegetarianos $\left(23,28 \mathrm{~kg} / \mathrm{m}^{2}\right)$, aun así ambos grupos se posicionaron en el rango considerado como normalidad $(18,5 \mathrm{~kg} /$ $\left.\mathrm{m}^{2}-24,99 \mathrm{~kg} / \mathrm{m}^{2}\right)$ por la Norma Oficial Mexicana NOM-043-SSA2-2012. Servicios básicos de salud. Promoción y educación para la salud en materia alimentaria. Criterios para brindar orientación para población mayor de 20 años $^{11}$. Según Key
T.J.10, los vegetarianos presentan un IMC de $1 \mathrm{~kg} / \mathrm{m}^{2}$ menor que la población que consume carne, lo que disminuye el riesgo de obesidad tanto en hombres como en mujeres mediante dietas vegetarianas, y por tanto el riesgo cardiovascular se ve reducido en comparación con las personas que consumen carne ${ }^{12}$. Además se ha comprobado que las personas vegetarianas tienen una menor concentración en los valores de colesterol de baja densidad y de la presión arterial ${ }^{4}$. Esta diferencia en parte se debe al alto consumo de fibra y al menor 
consumo de grasa animal en la dieta de los vegetarianos ${ }^{13}$.

En cuanto a la diferencia de medias en el porcentaje de masa grasa total de la población femenina, se obtuvo que entre la población ovolactovegetariana $(28,34 \%)$ y la vegana $(26,72 \%)$ no existe una diferencia significativa ( $p=0,41)$; ubicándose en el rango normal $(20,5-35,0 \%)$ de la MGT de acuerdo a la edad de 20 a 39 años y al sexo, según Gallagher $D^{14}$; lo mismo se observó en el género masculino al encontrándose en el rango normal (8,5 \%-21,2 $\%$ ), aunque los ovolactovegetarianos presentan más grasa corporal $(18,84 \%)$ que los veganos (13,86 \%).

En cuanto a la MMT, la población masculina ovolactovegetariana $(57,5 \mathrm{~kg})$ presenta más MMT que los veganos $(51,79$ $\mathrm{kg}$ ) siendo estadísticamente significativo ( $p$ $=0,03$ ). En la población femenina no se presentó una diferencia significativa si la persona es ovolactovegetariana o vegana; lo que explica que la tasa de cambio en el índice de MMT difiere entre los géneros, según Hull15.

De acuerdo a Morales L. ${ }^{16}$, en el estudio descriptivo transversal donde se evaluaron a 2167 personas cubanas de 15 a 97 años de edad, se tomó el rango de 20 a 29 años donde el percentil 50 tiene un valor para la MMT de $29,5 \mathrm{~kg}$ para hombres y 18,7 $\mathrm{kg}$ para mujeres. En el género femenino de los ovolactovegetarianos se obtuvo en la MMT un promedio de $39,49 \mathrm{~kg}$ y en el grupo de veganos un promedio de 40,19 $\mathrm{kg}$. En cuanto al género masculino se reportó un promedio en la MMT de 57,50 $\mathrm{kg}$ en ovolactovegetarianos, y un promedio de $51,79 \mathrm{~kg}$ en el grupo de veganos. Estos valores de MMT de cada grupo de vegetarianismo de acuerdo al género son mayores a los obtenidos por Morales L. ${ }^{16}$ debido probablemente a los estilos de vida de las poblaciones.

\section{Conclusiones}

Los ovolactovegetarianos del género masculino presentan mayor IMC, MGT y MMT que los veganos. Confirmando que existe una diferencia entre el grupo de los ovolactovegetarianos y el de los veganos para el género masculino en la composición corporal.

\section{Bibliografía}

1. Brignardello G.J., Heredia P.L., Paz Ocharán S.M., Durán A.S. Conocimientos alimentarios de vegetarianos y veganos chilenos. Rev. chil. nutr. [periódico na Internet]. 2013 Jun [citado 2014 Jun 13]; 40(2):129-134. Disponible en:

http://www.scielo.cl/scielo.php?script =sci arttext\&pid=S0717-75182013000200006\&lng =pt. h t t p : / / d . doi.org/10.4067/S0717 75182013000200006.

2. Unión Vegetariana Internacional. Preguntas más frecuentes: Definición (monografía); 2010 Abril 4 [citado 2014 Jun 7]. Disponible en: http://www.ivu.org/ spanish/faq/definitions.html.
3. Quiles L., Portolés O., Vicente J., Miquel M., Corella M.D. Efectos a corto plazo en la pérdida de peso de una dieta vegetariana baja en grasa. Nutrición Clínica y Dietética Hospitalaria. 2013; 33(3): 58-67. Disponible en: http://dialnet.unirioja.es/servlet/ ejemplar?codigo $=353493$.

4. Craig W.J., Mangels A.R.. Posición de la Asociación Americana de Dietética: Las dietas vegetarianas. Journal of the American Dietetic Association. January-February 2010; 14(1):10-26. Disponible en: http://www.sciencedirect.com/science/article/pii/ S1138032210700031.

5. Alvero J.R., Cabañas M.D., Herrero A., Martínez L., Moreno C., Porta J., et al. Protocolo de valoración de la composición corporal para el reconocimien- 
to médico-deportivo. Documento de Consenso del Grupo Español de Cineantropometría de la Federación Española de Medicina del Deporte Archivos de Medicina del Deporte. 2009; 26(131):166-179 Disponible en: http://www.femede.es/page.php?/ NumerosRevista/amd_2009_131.

6. Seon Yeong Lee and Dympnā Gallagher. Assessment methods in human body composition. National Institutes of Health. Curr Opin Clin Nutr Metab Care. Sep 2008; 11(5): 566-572. Disponible en: http://www. ncbi.nlm.nih.gov/pmc/articles/PMC2741386/.

7. Hull H.R., Thornton J., Wang J., Pierson R.N., Jr, $Z$ Kaleem, X Pi-Sunyer, et al. Fat-free mass index: changes and race/ethnic differences in adulthood. Int J Obes (Lond). Jan 2011; 35(1): 121-127. Disponible en: http://www.nature.com/ijo/journal/v35/ n1/full/ijo2010111a.html.

8. Martínez J.R., Villarino A.L., Polanco I., Iglesias C., Gil G., Ramos C., et al. Recomendaciones de bebida e hidratación para la población española. Nutrición Clínica y Dietética Hospitalaria. 2008; 28(2): 3-19.

9. Pérez Miguelsanz M.J., Cabrera Parra W., Varela Moreiras G., Garaulet M. Distribución regional de la grasa corporal: Uso de técnicas de imagen como herramienta de diagnóstico nutricional. Nutr. Hosp. [revista en la Internet]. 2010 Abr [citado 2014 Jun 11]; 25(2): 207-223. Disponible en: http://scielo. isciii.es/scielo.php?script $=$ sci_arttext $\&$ pid $=$ S0212 $16112010000200003 \& \operatorname{lng}=\mathrm{es}$.

10. Key T.J., Davey G.K., Appleby P.N. Health benefits of a vegetarian diet. Proceedings of the Nutrition Society. Cambridge Journals. 1999 April; 58(2): 271275 .
11. Diario Oficial de la Federación. Norma oficial Mexicana NOM-043-SSA2-2012, Servicios básicos de salud. Promoción y educación para la salud en materia alimentaria. Criterios para brindar orientación. [Fecha de actualización 2013 Ene 22; citado 2014 Jun 09].

12. Fernandes K., De Arruda C., Flores H., Dos Santos SK., Pereira L. Estado nutricional, estilo de vida y riesgo cardiovascular de ovolactovegetarianos e omnívoros. Archivos latinoamericanos de nutrición. 2010; 60 (3): $220-226$.

13. Couceiro P., Slywitch E., Lenz F. Padrão alimentar da dieta vegetariana. Einstein. 2008; 6(3):365-73. Disponible en:

http://www.researchgate.net/publication/26572246 Eating pattern of vegetarian diet.

14. Gallagher D., Hemsfield S.B., Heo M., Jebb S., Murgatroyd $P$., Sakamoto Y. Body mass index guidelines: corresponding \% fat standards based on three-country study. 1999. Disponible en: http://www.tanita. com/en/supporting-research/p1/.

15. Hull H.R., Thornton J., Wang J., Pierson R.N., Jr, $Z$ Kaleem, X Pi-Sunyer, et al. Fat-free mass index: changes and race/ethnic differences in adulthood. Int J Obes (Lond). Jan 2011; 35(1): 121-127. Disponible en: http://www.nature.com/ijo/journal/v35/ n1/full/ijo2010111a.html.

16. Morales L., Román M., Núñez B., Lara L., Marañón C., Castillo B. Composición corporal: intervalos de lo normal en el estudio mediante bioimpedancia eléctrica de una población de referencia. MEDISAN. 2004; 8(4): 22-34. 